\title{
Estimation of Newly Established Iterative Scheme for Generalized Nonexpansive Mappings
}

\author{
Aftab Hussain ${ }^{D},{ }^{1}$ Nawab Hussain $\left(\mathbb{D},{ }^{1}\right.$ and Danish $\mathrm{Ali}^{2}$ \\ ${ }^{1}$ Department of Mathematics, King Abdulaziz University, P.O. Box 80203 Jeddah 21589, Saudi Arabia \\ ${ }^{2}$ Department of Mathematics, Faculty of Natural Science, Khawaja Fareed University of Engineering and Technology, \\ 64100 Rahim Yar Khan, Pakistan
}

Correspondence should be addressed to Aftab Hussain; aniassuirathak@kau.edu.sa and Nawab Hussain; nhusain@kau.edu.sa

Received 17 October 2020; Revised 25 December 2020; Accepted 11 February 2021; Published 11 March 2021

Academic Editor: Maria Alessandra Ragusa

Copyright (C) 2021 Aftab Hussain et al. This is an open access article distributed under the Creative Commons Attribution License, which permits unrestricted use, distribution, and reproduction in any medium, provided the original work is properly cited.

We introduce a new iterative method in this article, called the $D$ iterative approach for fixed point approximation. Analytically, and also numerically, we demonstrate that our established D I.P is faster than the well-known I.P of the prior art. Finally, in a uniformly convex Banach space environment, we present weak as well as strong convergence theorems for Suzuki's generalized nonexpansive maps. Our findings are an extension, refinement, and induction of several existing iterative literatures.

\section{Introduction and Preliminaries}

In many branches of mathematics and various sciences, the existence of a fixed point is crucial. A completely fine synthesis of analysis, geometry, and topology is the fixed point theory. In particular, fixed point techniques are used in economics, biology, engineering, biochemistry, game theory, and physics. So, once the presence of a fixed point is found, it is difficult to find its value; that is why we use an I.P to calculate them. A variety of I.Ps have been introduced and it is not possible to discuss all I.Ps. The famous Banach contraction theorem uses the Picard I.P to approximate fixed points. Other well-known I.Ps can be found in references [1-8, 1518]. The approximation speed of the I.P has a major role, and an I.P tends to be chosen in another iterative process. In [2], the writer believes that the approximation rate of the Agarwal I.P is like that of the Picard I.P and is faster than the contraction mapping of the Mann I.P. In [9], the writers help by numerical examples to prove for nonexpansive mapping, that the approximation rate of the Picard-S I.P is better than existing literature. They proved that the convergence rate was good. In [6], the creators illustrate that the approximation rate of the $M^{*}$ I.P is better than existing literature. Recently, in [7], another I.P, namely, M I.P, was developed, and it is shown that its approximation rate is better than existing literature. In [10], one more I.P called $K$ I.P was developed, in which they have shown that the approximation rate is better than previous literature. By hypothesis, we introduce a new I.P, namely, the $D$ iterative process.

Numerically, the approximation rate for the latest I.P is contrasted with the Agarwal I.P, Picard-S I.P, $M$ I.P, $M^{*}$ I.P, and $K$ I.P. We present the weak as well as strong convergence theorems of Suzuki generalized nonexpansive maps and contraction map for our newly developed I.P. We first remember those definitions, ideas, and lemmas which we have to use in the upcoming two sections. A Banach space $X$ is referred to as uniformly convex [11] if $\forall \varepsilon \in(0,2] \exists \delta>0$ s.t for $\xi, \eta \in X$,

$$
\left.\begin{array}{l}
\|\xi\| \leq 1 \\
\|\eta\| \leq 1 \leq 1 \\
\|\xi-\eta\|>\varepsilon>\varepsilon
\end{array}\right\} \Rightarrow\left\|\frac{\xi+\eta}{2}\right\| \leq \delta .
$$

$X$ is referred to obey the Opial property [4] if $\forall\left\{\xi_{n}\right\} \in X$, approaching weakly to $\xi \in X$, we have 


$$
\begin{gathered}
\lim _{n \rightarrow \infty} \sup \left\|\xi_{n}-\xi\right\|<\lim _{n \rightarrow \infty} \sup \left\|\xi_{n}-\eta\right\|, \\
\forall \eta \in X \text { s.t } \eta \neq \xi .
\end{gathered}
$$

If $F(p)=p, T(F)$ denotes the set of all fixed points of $F$; a point $p$ is referred to as the fixed point of mapping $F$.

Let $C \neq \phi \subseteq X$, and $F: C \rightarrow C$ is referred as contraction if $\exists \beta \in(0,1)$ s.t $\|F \xi-F \eta\| \leq \beta\|\xi-\eta\|, \forall \xi, \eta \in C$.

$F: C \rightarrow C$ is referred to as nonexpansive if $\|F \xi-F \eta\| \leq \|$ $\xi-\eta \| \forall \xi, \eta \in C$, and quasinonexpansive if $\forall \xi \in C$ and $p \in T(F$ ), so $\|F \xi-p\| \leq\|\xi-p\|$. In [12], $F: C \rightarrow C$ is referred to satisfy condition $(M)$ if $\forall \xi, \eta \in C$, we have

$$
\frac{1}{2}\|\xi-F \xi\| \leq\|\xi-\eta\| \Rightarrow\|F \xi-F \eta\| \leq\|\xi-\eta\|
$$

In [12], the author approved that the mapping holding condition $(M)$ is weaker than the nonexpansive mapping and stronger than the quasinonexpansive mapping. The mapping which holds condition $(M)$ is called the Suzuki generalized nonexpansive mapping. Reference [12] includes the fixed point theorem and convergence theorem for Suzuki's generalized nonexpansive mapping. Recently, the fixed point theorem of the Suzuki generalized nonexpansive mapping has been studied by many authors [5-7]. Now, we define properties of Suzuki generalized nonexpansive mappings.

Proposition 1. Let $C \neq \phi \subseteq X$ and $F: C \rightarrow C$. Then,

(i) ([12], Proposition 1) If F is nonexpansive, so F must be a Suzuki generalized nonexpansive

(ii) ([12], Proposition 2) If F is a Suzuki generalized nonexpansive having a fixed point, then $F$ must be a quasinonexpansive

(iii) ([12], Lemma 7) If $F$ is a Suzuki generalized nonexpansive, then $\left\|\xi-F_{\eta}\right\| \leq 3\|F \xi-\xi\|+\|\xi-\eta\| \forall \xi, \eta \in C$.

Lemma 2 ([12], Proposition 3). Let $F: C \rightarrow C$ which satisfies the Opial property. Suppose that $F$ is a Suzuki generalized nonexpansive. If $\left\{\xi_{n}\right\}$ approaches weakly to $t$ and $\lim _{n \rightarrow \infty} \| F$ $\xi_{n}-\xi_{n} \|=0$, then $F t=t$. So, $I-F$ is demiclosed at zero.

Lemma 3 ([12], Theorem 5). Let $F: C \rightarrow C$ and $F$ is a Suzuki generalized nonexpansive. Then $F$ must have a fixed point.

Lemma 4 ([13], Lemma 1.3). Let $\left\{t_{n}\right\}$ be any real sequence in $X$ s.t $0<p \leq t_{n} \leq q<1 \forall n \geq 1$. Let $\left\{\xi_{n}\right\}$ and $\left\{\eta_{n}\right\}$ be any two sequences of $X$ s.t limsup $p_{n \rightarrow \infty}\left\|\xi_{n}\right\| \leq r$, limsup $p_{n \rightarrow \infty}\left\|\eta_{n}\right\| \leq r$, and $\limsup _{n \rightarrow \infty}\left\|t_{n} \xi_{n}+\left(1-t_{n}\right) \eta_{n}\right\|=r$ hold for some $r \geq 0$. Then, $\lim _{n \rightarrow \infty}\left\|\xi_{n}-\eta_{n}\right\|=0$.

Let $C \neq \phi \subseteq X$, and let $\left\{\xi_{n}\right\}$ be a bounded sequence $\in X$. For $\xi \in X$, we set

$$
r\left(\xi,\left\{\xi_{n}\right\}\right)=\limsup _{n \rightarrow \infty}\left\|\xi_{n}-\xi\right\|
$$

The asymptotic radius of $\left\{\xi_{n}\right\}$ relative to $C$ is given by

$$
r\left(C,\left\{\xi_{n}\right\}\right)=\inf \left\{r\left(\xi,\left\{\xi_{n}\right\}\right): \xi \in C\right\},
$$

and the asymptotic center of $\left\{\xi_{n}\right\}$ relative to $C$ is defined as

$$
A\left(C,\left\{\xi_{n}\right\}\right)=\left\{\xi \in C: r\left(\xi,\left\{\xi_{n}\right\}\right)=r\left(C,\left\{\xi_{n}\right\}\right) .\right.
$$

Definition 5 (see [2]). Let $\left\{\xi_{n}\right\}_{n=0}^{\infty}$ and $\left\{\eta_{n}\right\}_{n=0}^{\infty}$ be two fixed point I.P sequences that approach the single fixed point $p$ and $\left\|\xi_{n}-p\right\| \leq u_{n}$ and $\left\|\eta_{n}-p\right\| \leq v_{n}, \forall n \geq 0$. If the sequence $\left\{u_{n}\right\}_{n=0}^{\infty}$ and $\left\{v_{n}\right\}_{n=0}^{\infty}$ approaches to $u$ and $v$,respectively, and $\lim _{n \rightarrow \infty}\left\|u_{n}-u\right\| /\left\|v_{n}-v\right\|=0$, then $\left\{\xi_{n}\right\}_{n=0}^{\infty}$ approaches faster as $\left\{\eta_{n}\right\}_{n=0}^{\infty}$ to $p$.

\section{The $D$ Iteration Process}

In this section, let $n \geq 0$ and $\left\{\theta_{n}\right\}$ and $\left\{\vartheta_{n}\right\}$ are real sequences $\in[0,1]$ and $C \neq \phi \subseteq X$. For detail on I.P, please see [14].

Following is two step Agrawal I.P or S I.P as

$$
\left\{\begin{array}{l}
\xi_{0} \in C, \\
\eta_{n}=\left(1-\vartheta_{n}\right) \xi_{n}+\vartheta_{n} F \xi_{n} \\
\xi_{n+1}=\left(1-\theta_{n}\right) F \xi_{n}+\theta_{n} F \eta_{n} .
\end{array}\right.
$$

In [9], the authors develop a new I.P called Picard-S I.P as given below:

$$
\left\{\begin{array}{l}
\xi_{0} \in C, \\
\omega_{n}=\left(1-\vartheta_{n}\right) \xi_{n}+\vartheta_{n} F \xi_{n}, \\
\eta_{n}=\left(1-\theta_{n}\right) F \xi_{n}+\theta_{n} T \omega_{n}, \\
\xi_{n+1}=F \eta_{n} .
\end{array}\right.
$$

They have demonstrated that with Picard-S I.P, we approximate the fixed point of contraction mapping. Also, by a numerical example, they proved that the Picard-S I.P have a better approximation rate than all previously developed I.P.

In [7], the authors introduced a new I.P, namely, $M$ I.P, which is given below:

$$
\left\{\begin{array}{l}
\xi_{0} \in C, \\
\omega_{n}=\left(1-\theta_{n}\right) \xi_{n}+\theta_{n} F \xi_{n}, \\
\eta_{n}=F \omega_{n}, \\
\xi_{n+1}=F \eta_{n} .
\end{array}\right.
$$

In [6], the authors introduced one new I.P, namely, $M^{*}$ I.P, which is defined as 


$$
\left\{\begin{array}{l}
\xi_{0} \in C, \\
\omega_{n}=\left(1-\vartheta_{n}\right) \xi_{n}+\vartheta_{n} F \xi_{n}, \\
y_{n}=F\left(\left(1-\theta_{n}\right) \xi_{n}+\theta_{n} F \omega_{n}\right), \\
\xi_{n+1}=F \eta_{n} .
\end{array}\right.
$$

They proved that each of (10) and (11) is moving faster as compared to (8) and (9).

In [10], the authors introduced a three-step I.P, called $K$ I.P, which is given below:

$$
\left\{\begin{array}{l}
\xi_{0} \in C, \\
\omega_{n}=\left(1-\vartheta_{n}\right) \xi_{n}+\vartheta_{n} F \xi_{n}, \\
\eta_{n}=F\left(\left(1-\theta_{n}\right) F \xi_{n}+\theta_{n} F \omega_{n}\right), \\
\xi_{n+1}=F \eta_{n} .
\end{array}\right.
$$

They insisted that the new I.P converged quickly. By providing an example, they showed that the $K$ I.P has a better approximating rate than the S I.P., Picard-S I.P, $M$ I.P, and $M^{*}$ I.P.

In this competition, we developed following (new) threestep I.P, namely, D I.P, defined by

$$
\left\{\begin{array}{l}
\xi_{0} \in C, \\
\omega_{n}=F\left(\left(1-\vartheta_{n}\right) \xi_{n}+\vartheta_{n} F \xi_{n}\right), \\
\eta_{n}=F\left(\left(1-\theta_{n}\right) F \xi_{n}+\theta_{n} F \omega_{n}\right), \\
\xi_{n+1}=F \eta_{n} .
\end{array}\right.
$$

In order to prove that our new I.P (13) have a better approximation rate as compared to (8),(9), (10), (11), and (12), first, we generally prove that our I.P strongly converges to unique fixed point, and then it is supported with a numerical example.

Theorem 6. Let the contraction map $F: C \rightarrow C$, where $C \neq$ $\phi \subseteq X$. Assume $\left\{\xi_{n}\right\}_{n=0}^{\infty}$ to be an iterative sequence generated by D I.P, where $\left\{\theta_{n}\right\}_{n=0}^{\infty}$ and $\left\{\vartheta_{n}\right\}_{n=0}^{\infty} \in\left[\begin{array}{ll}0 & 1\end{array}\right]$ are real sequences satisfying $\sum_{n=0}^{\infty} \theta_{n}=\infty$ or $\sum_{n=0}^{\infty} \vartheta_{n}=\infty$. Then, $\left\{\xi_{n}\right\}_{n=0}^{\infty}$ approaches strongly to a unique fixed point of $F$.

Proof. As $F$ is a contraction in $X$, so $F$ must have a unique fixed point in $C$. Assume $p$ is a particular fixed point of $F$. From D I.P, we get

$$
\begin{aligned}
\left\|\omega_{n}-p\right\| & =\left\|F\left(\left(1-\vartheta_{n}\right) \xi_{n}+\vartheta_{n} F \xi_{n}\right)-F p\right\| \\
& \leq k\left\|\left(1-\vartheta_{n}\right) \xi_{n}+\vartheta_{n} F \xi_{n}-p\right\| \\
& \leq k\left\|\left(1-\vartheta_{n}\right)\left(\xi_{n}-p\right)+\beta_{n}\left(F \xi_{n}-p\right)\right\| \\
& \leq k\left(1-\vartheta_{n}\right)\left\|\xi_{n}-p\right\|+\vartheta_{n}\left\|F \xi_{n}-p\right\| \\
& \leq k\left\{\left(1-\vartheta_{n}\right)\left\|\xi_{n}-p\right\|+k \vartheta_{n}\left\|\xi_{n}-p\right\|\right. \\
& \leq k\left\{1-\vartheta_{n}(1-k)\right\}\left\|\xi_{n}-p\right\| .
\end{aligned}
$$

Now,

$$
\begin{aligned}
\left\|\eta_{n}-p\right\| & =\left\|F\left(\left(1-\theta_{n}\right) F \xi_{n}+\theta_{n} F \omega_{n}\right)-F p\right\| \\
& \leq k\left[\left(1-\theta_{n}\right)\left\|F \xi_{n}-p\right\|+\theta_{n}\left\|F \omega_{n}-p\right\|\right] \\
& \leq k\left[\left(1-\theta_{n}\right) k\left\|\xi_{n}-p\right\|+\theta_{n} k\left\|\omega_{n}-p\right\|\right] \\
& \leq k^{2}\left[\left(1-\theta_{n}\right)\left\|\left(\xi_{n}-p\right)\right\|+\theta_{n}\left\|\omega_{n}-p\right\|\right] \\
& \leq k^{2}\left[\left(1-\theta_{n}\right)\left\|\left(\xi_{n}-p\right)\right\|+\theta_{n}\left(k\left\{1-\vartheta_{n}(1-k)\right\}\left\|\xi_{n}-p\right\|\right)\right. \\
& \leq k^{2}\left[1-\left(\theta_{n}+k \theta_{n} \vartheta_{n}\right)(1-k)\right]\left\|\left(\xi_{n}-p\right)\right\| .
\end{aligned}
$$

Then,

$$
\begin{aligned}
\left\|\xi_{n+1}-p\right\| & =\left\|F \eta_{n}-F p\right\| \leq k\left\|\eta_{n}-p\right\| \\
& \leq k^{3}\left[1-\left(\theta_{n}+k \theta_{n} \vartheta_{n}\right)(1-k)\right]\left\|\left(\xi_{n}-p\right)\right\| .
\end{aligned}
$$

After repetition, we get

$$
\begin{gathered}
\left\|\xi_{n}-p\right\| \leq k^{3}\left[1-\left(\theta_{n-1}+k \theta_{n-1} \vartheta_{n-1}\right)(1-k)\right]\left\|\left(\xi_{n-1}-p\right)\right\| \\
\left\|\xi_{n-1}-p\right\| \leq k^{3}\left[1-\left(\theta_{n-2}+k \theta_{n-2} \vartheta_{n-2}\right)(1-k)\right]\left\|\left(\xi_{n-2}-p\right)\right\| \\
\vdots \\
\left\|\xi_{n-2}-p\right\| \leq k^{3}\left[1-\left(\theta_{n-3}+k \theta_{n-3} \vartheta_{n-3}\right)(1-k)\right]\left\|\left(\xi_{n-3}-p\right)\right\| \\
\left\|\xi_{1}-p\right\| \leq k^{3}\left[1-\left(\theta_{0}+k \theta_{0} \vartheta_{0}\right)(1-k)\right] \|\left(\xi_{0}-p\right) .
\end{gathered}
$$

Therefore, we obtain $\left\|\xi_{n+1}-p\right\| \leq k^{3(n+1)}\left\|\left(\xi_{0}-p\right)\right\| \prod_{i=0}^{n}[1$ $\left.-\left(\theta_{i}+k \theta_{i} \vartheta_{i}\right)(1-k)\right]$. Now, $k<1$ so $(1-k)>0$ and $\theta_{n}, \vartheta_{n} \leq$ $1 \forall n \in N$. Thus, we get $\left[1-\left(\theta_{i}+k \theta_{i} \vartheta_{i}\right)(1-k)\right]<1$ and $\forall n \in$ $N$. As $1-\mathrm{x} \leq e^{-x}, \forall \xi \in\left[\begin{array}{ll}0 & 1\end{array}\right]$. So we have

$$
\left\|\xi_{n+1}-p\right\| \leq k^{3(n+1)}\left\|\left(\xi_{0}-p\right)\right\| e^{-(1-k)} \sum_{i=0}^{n}\left\{\theta_{i}+k \theta_{i} \vartheta_{i}\right\} .
$$

Taking the limits $n \rightarrow \infty$ both sides, we get $\lim _{n \rightarrow \infty} \| \xi_{n}$ $-p \|=0$.

Theorem 7. Let $C \neq \phi \subseteq X$, and let contraction map $F: C$ $\rightarrow C$ holding condition $(M)$, having a fixed point $p$. For a given $\xi_{0}=\eta_{0}$, let $\left\{\xi_{n}\right\}_{n=0}^{\infty}$ and $\left\{\eta_{n}\right\}_{n=0}^{\infty}$ be iterative sequences developed by D I.P and Picard-S I.P as in [11], respectively, where $\left\{\theta_{n}\right\}_{n=0}^{\infty}, \quad\left\{\vartheta_{n}\right\}_{n=0}^{\infty}$, and $\left\{\eta_{n}\right\}_{n=0}^{\infty} \in[0 ; 1]$ are real sequences satisfying $\theta \leq \theta_{n}<1$ and $\vartheta \leq \vartheta_{n}<1$, for some $\theta, \vartheta$ $>0$ and $\forall n \in N$. Then, $\left\{\xi_{n}\right\}_{n=0}^{\infty}$ approaches to $p$ firstly rather than $\left\{\eta_{n}\right\}_{n=0}^{\infty}$.

Proof. By Theorem 2.5 in [11], we have

$$
\left\|\eta_{n+1}-p\right\| \leq k^{2(n+1)}\left\|\left(\eta_{0}-p\right)\right\| \prod_{i=0}^{n}\left\{1-\theta_{i} \vartheta_{i}(1-k)\right\} .
$$


Since $\theta \leq \theta_{n}$ and for all $n \in \mathrm{N}$, we obtain $\left\|\eta_{n+1}-p\right\| \leq$ $k^{2(n+1)}\left\|\left(\eta_{0}-p\right)\right\|\left\{1-\theta_{i} \vartheta_{i}(1-k)\right\}^{n+1}$. $=k^{2(n+1)}\left\|\left(\eta_{0}-p\right)\right\|\left\{1-\theta_{i} \vartheta_{i}(1-k)\right\}^{n+1}$.

Now, from Theorem 6 , we get

$$
\left\|\xi_{n+1}-p\right\| \leq k^{3(n+1)}\left\|\left(\xi_{0}-p\right)\right\| \prod_{i=0}^{n}\left\{1-\left(\theta_{i}+k \theta_{i} \vartheta_{i}\right)(1-k)\right\}
$$

Again $\theta \leq \theta_{n}$ for all $n \in N$ gives

$$
\left\|\xi_{n+1}-p\right\| \leq k^{3(n+1)}\left\|\left(\xi_{0}-p\right)\right\|\left\{1-\left(\theta_{i}+k \theta_{i} \vartheta_{i}\right)(1-k)\right\}^{n+1} .
$$

Let $\quad b_{n}=k^{3(n+1)}\left\|\left(\xi_{0}-p\right)\right\|\left\{1-\left(\theta_{i}+k \theta_{i} \vartheta_{i}\right)(1-k)\right\}^{n+1}$. Then,

$$
\frac{b_{n}}{a_{n}}=\frac{k^{3(n+1)}\left\|\left(\xi_{0}-p\right)\right\|\left\{1-\left(\theta_{i}+k \theta_{i} \vartheta_{i}\right)(1-k)\right\}^{n+1}}{k^{2(n+1)}\left\|\left(\eta_{0}-p\right)\right\|\left\{1-\theta_{i} \vartheta_{i}(1-k)\right\}} .
$$

Thus, taking the limit as $n \rightarrow \infty \lim _{n \rightarrow \infty}\left(b_{n} / a_{n}\right)=0$. Hence, the result follows.

Next, we present a result which defines the better approximation rate between the $D$ and $M$ I.P [7].

Theorem 8. Let $C \neq \phi \subseteq X$, also let contraction map $F: C$ $\rightarrow C$ holding condition $(M)$, having a fixed point $p$. For a given $\xi_{0}=\eta_{0}$, let $\left\{\xi_{n}\right\}_{n=0}^{\infty}$ and $\left\{u_{n}\right\}_{n=0}^{\infty}$ be iterative sequences developed by D I.P and M I.P as in [7], respectively, where $\left\{\theta_{n}\right\}_{n=0}^{\infty},\left\{\vartheta_{n}\right\}_{n=0}^{\infty}$, and $\left\{\eta_{n}\right\}_{n=0}^{\infty} \in[0 ; 1]$ are real sequences satisfying $\theta \leq \theta_{n}<1$ and $\vartheta \leq \vartheta_{n}<1$, for some $\theta, \vartheta>0$ and $\forall n \in$ $N$. Then, $\left\{\xi_{n}\right\}_{n=0}^{\infty}$ approaches to $p$ firstly rather than $\left\{u_{n}\right\}_{n=0}^{\infty}$

Proof. By Theorem 6, we have

$$
\left\|\xi_{n+1}-p\right\| \leq k^{3(\mathrm{n}+1)}\left\|\left(\xi_{0}-p\right)\right\| \prod_{i=0}^{n}\left\{1-\left(\theta_{i}+k \theta_{i} \vartheta_{i}\right)(1-k)\right\}
$$

Since $\theta \leq \theta_{n}$ and for all $n \in N$, we obtain

$$
\left\|\xi_{n+1}-p\right\| \leq k^{3(n+1)}\left\|\left(\xi_{0}-p\right)\right\|\left\{1-\left(\theta_{i}+k \theta_{i} \vartheta_{i}\right)(1-k)\right\}^{n+1}
$$

Let $\quad a_{n}=k^{3(n+1)}\left\|\left(\xi_{0}-p\right)\right\|\left\{1-\left(\theta_{i}+k \theta_{i} \vartheta_{i}\right)(1-k)\right\}^{n+1}$. Then, for the $M$ iteration, we have

$$
\begin{aligned}
& \left\{\begin{array}{l}
u_{0} \in C, \\
w_{n}=\left(1-\vartheta_{n}\right) u_{n}+\vartheta_{n} F u_{n}, \\
v_{n}=F w_{n}, \\
u_{n+1}=F v_{n},
\end{array}\right. \\
& \left\|w_{n}-p\right\|=\left\|\left(\left(1-\vartheta_{n}\right) u_{n}+\vartheta_{n} F u_{n}\right)-F p\right\| \\
& \leq\left\|\left(1-\vartheta_{n}\right) u_{n}+\vartheta_{n} F u_{n}-p\right\| \\
& \leq\left\|\left(1-\vartheta_{n}\right)\left(u_{n}-p\right)+\vartheta_{n}\left(F u_{n}-p\right)\right\| \\
& \leq\left(1-\vartheta_{n}\right)\left\|\left(u_{n}-p\right)\right\|+\vartheta_{n}\left\|\left(F u_{n}-p\right)\right\| \\
& \leq\left\{\left(1-\vartheta_{n}\right)\left\|\left(u_{n}-p\right)\right\|+k \vartheta_{n}\left\|\left(u_{n}-p\right)\right\|\right\} \\
& \leq k\left\{1-\vartheta_{n}(1-k)\right\}\left\|\left(u_{n}-p\right)\right\| \text {. }
\end{aligned}
$$

Now,

$$
\begin{aligned}
\left\|v_{n}-p\right\| & \leq\left\|F w_{n}-p\right\| \leq k\left\|w_{n}-p\right\| \\
& \leq k\left\{1-\vartheta_{n}(1-k)\right\}\left\|\left(u_{n}-p\right)\right\| .
\end{aligned}
$$

Therefore, we get $\left\|u_{n+1}-p\right\| \leq\left\|F v_{n}-p\right\| \leq k\left\|v_{n}-p\right\| \leq \mathrm{k}^{2}\{1$ $\left.-\vartheta_{n}(1-k)\right\}\left\|\left(u_{n}-p\right)\right\|$.

After repetition,

$$
\begin{gathered}
\left\|u_{n}-p\right\| \leq k^{2}\left\{1-\vartheta_{n-1}(1-k)\right\}\left\|\left(u_{n-1}-p\right)\right\|, \\
\left\|u_{n-1}-p\right\| \leq k^{2}\left\{1-\vartheta_{n-2}(1-k)\right\}\left\|\left(u_{n-2}-p\right)\right\|, \\
\left\|u_{n-2}-p\right\| \leq k^{2}\left\{1-\vartheta_{n-3}(1-k)\right\}\left\|\left(u_{n-3}-p\right)\right\|, \\
\vdots \\
\left\|u_{1}-p\right\| \leq k^{2}\left\{1-\vartheta_{0}(1-k)\right\}\left\|\left(u_{0}-p\right)\right\| .
\end{gathered}
$$

Thus, we have $\left\|u_{n+1}-p\right\| \leq k^{2(n+1)}\left\|\left(u_{0}-p\right)\right\| \prod_{i=0}^{n}\left\{1-\vartheta_{i}(\right.$ $1-k)\}$. Now, since $\vartheta \leq \vartheta_{n}$ and for all $n \in N$, we obtain $\| u_{n+1}$ $-p\left\|\leq k^{2(n+1)}\right\|\left(u_{0}-p\right) \|\left\{1-\vartheta \theta_{i}(1-k)\right\}^{n+1}$. Let $b_{n}=k^{2(n+1)} \|($ $\left.u_{0}-p\right) \|\left\{1-\vartheta \theta_{i}(1-k)\right\}^{n+1}$.

Then,

$$
\frac{a_{n}}{b_{n}}=\frac{k^{3(n+1)}\left\|\left(\xi_{0}-p\right)\right\|\left\{1-\left(\theta_{i}+k \theta_{i} \vartheta_{i}\right)(1-k)\right\}^{n+1}}{k^{2(n+1)}\left\|\left(u_{0}-p\right)\right\|\left\{1-\vartheta \theta_{i}(1-k)\right\}^{n+1}} .
$$

Thus, taking the limit as $n \rightarrow \infty \lim _{n \rightarrow \infty}\left(b_{n} / a_{n}\right)=0$. Hence, the result follows. [6].

Next, we prove that $D$ I.P is faster than that of the $M^{*}$ I.P

Theorem 9. Let $C \neq \phi \subseteq X$; also, let a contraction mapping $F$ $: C \rightarrow C$ holding condition $(M)$, having a particular fixed point $p$. For a given $\xi_{0}=\eta_{0}$, let $\left\{\xi_{n}\right\}_{n=0}^{\infty}$ and $\left\{u_{n}\right\}_{n=0}^{\infty}$ be iterative sequences developed by D I.P and M I.P as in [6], respectively, where $\left\{\theta_{n}\right\}_{n=0}^{\infty},\left\{\vartheta_{n}\right\}_{n=0}^{\infty}$, and $\left\{\eta_{n}\right\}_{n=0}^{\infty} \in[0 ; 1]$ are real sequences satisfying $\theta \leq \theta_{n}<1$ and $\vartheta \leq \vartheta_{n}<1$, for some $\theta$, $\vartheta$ $>0$ and $\forall n \in N$. Then, $\left\{\xi_{n}\right\}_{n=0}^{\infty}$ approaches to $p$ firstly rather than $\left\{u_{n}\right\}_{n=0}^{\infty}$. 
Proof. By result 2.1, we have

$$
\left\|\xi_{n+1}-p\right\| \leq k^{3(n+1)}\left\|\left(\xi_{0}-p\right)\right\| \prod_{i=0}^{n}\left\{1-\left(\theta_{i}+k \theta_{i} \vartheta_{i}\right)(1-k)\right\}
$$

Since $\theta \leq \theta_{n}$ and for all $n \in N$, we obtain

$$
\left\|\xi_{n+1}-p\right\| \leq k^{3(n+1)}\left\|\left(\xi_{0}-p\right)\right\|\left\{1-\left(\theta_{i}+k \theta_{i} \vartheta_{i}\right)(1-k)\right\}^{n+1}
$$

Let $\quad a_{n}=k^{3(n+1)}\left\|\left(\xi_{0}-p\right)\right\|\left\{1-\left(\theta_{i}+k \theta_{i} \vartheta_{i}\right)(1-k)\right\}^{n+1}$. Then, for the $M^{*}$ iteration, we have

$$
\begin{aligned}
\left\{\begin{array}{l}
u_{0} \in C, \\
w_{n}=\left(1-\vartheta_{n}\right) u_{n}+\vartheta_{n} F u_{n}, \\
v_{n}=F\left(\left(1-\theta_{n}\right) u_{n}+\theta_{n} F w_{n}\right), \\
u_{n+1}=F v_{n},
\end{array}\right. \\
\left\|w_{n}-p\right\|=\left\|\left(\left(1-\vartheta_{n}\right) u_{n}+\vartheta_{n} F u_{n}\right)-F p\right\| \\
\leq\left\|\left(1-\vartheta_{n}\right) u_{n}+\vartheta_{n} F u_{n}-p\right\| \\
\leq\left\|\left(1-\vartheta_{n}\right)\left(u_{n}-p\right)+\vartheta_{n}\left(F u_{n}-p\right)\right\| \\
\leq\left(1-\vartheta_{n}\right)\left\|\left(u_{n}-p\right)\right\|+\vartheta_{n}\left\|\left(F u_{n}-p\right)\right\| \\
\leq\left\{\left(1-\vartheta_{n}\right)\left\|\left(u_{n}-p\right)\right\|+k \vartheta_{n}\left\|\left(u_{n}-p\right)\right\|\right\} \\
\leq\left\{1-\vartheta_{n}(1-k)\right\}\left\|\left(u_{n}-p\right)\right\| .
\end{aligned}
$$

Now,

$$
\begin{aligned}
\left\|v_{n}-p\right\| & =\left\|F\left(\left(1-\theta_{n}\right) u_{n}+\theta_{n} F w_{n}\right)-F p\right\| \\
& \leq k\left\|\left(1-\theta_{n}\right) u_{n}+\theta_{n} F w_{n}-p\right\| \\
& \leq k\left\|\left(1-\theta_{n}\right)\left(u_{n}-p\right)+\theta_{n}\left(F w_{n}-p\right)\right\| \\
& \leq k\left(1-\theta_{n}\right)\left\|\left(u_{n}-p\right)\right\|+\theta_{n}\left\|\left(F w_{n}-p\right)\right\| \\
& \leq k\left\{\left(1-\theta_{n}\right)\left\|\left(u_{n}-p\right)\right\|+k \theta_{n}\left\|\left(w_{n}-p\right)\right\|\right\} \\
& \leq k\left\{\left(1-\theta_{n}\right)\left\|\left(u_{n}-p\right)\right\|+k \theta_{n}\left\{1-\vartheta_{n}(1-k)\right\}\left\|\left(u_{n}-p\right)\right\|\right\} \\
& \leq k\left\{\left(1-\theta_{n}\right)+k \theta_{n}-k \theta_{n} \vartheta_{n}(1-k)\right\}\left\|\left(u_{n}-p\right)\right\| \\
& \leq k\left\{\left(1-(1-k) \theta_{n}-k \theta_{n} \vartheta_{n}(1-k)\right\}\left\|\left(u_{n}-p\right)\right\|\right. \\
& \leq k\left\{\left(1-\theta_{n}(1-k)\left(1-k \vartheta_{n}\right)\right\}\left\|\left(u_{n}-p\right)\right\|\left\|u_{n+1}-p\right\|\right. \\
& \leq\left\|F v_{n}-p\right\| \leq k^{2}\left\{\left(1-\theta_{n}(1-k)\left(1-k \vartheta_{n}\right)\right\}\left\|\left(u_{n}-p\right)\right\| .\right.
\end{aligned}
$$

After repetition,

$$
\begin{gathered}
\left\|u_{n}-p\right\| \leq k^{2}\left\{1-\vartheta_{n-1}(1-k)\right\}\left\|\left(u_{n-1}-p\right)\right\|, \\
\left\|u_{n-1}-p\right\| \leq k^{2}\left\{1-\vartheta_{n-2}(1-k)\right\}\left\|\left(u_{n-2}-p\right)\right\|, \\
\left\|u_{n-1}-p\right\| \leq k^{2}\left\{1-\vartheta_{n-2}(1-k)\right\}\left\|\left(u_{n-2}-p\right)\right\|, \\
\vdots \\
\left\|u_{1}-p\right\| \leq k^{2}\left\{1-\vartheta_{0}(1-k)\right\}\left\|\left(u_{0}-p\right)\right\| .
\end{gathered}
$$

TABle 1: Sequence generated by $D, K, M^{*}, M$, Picard-S, and $S$ I.P for mapping $F$ of Example 10.

\begin{tabular}{lcccccc}
\hline & $S$ & Picard-S & $M$ & $M^{*}$ & $K$ & $D$ \\
\hline$\xi_{0}$ & 3.5 & 3.5 & 3.5 & 3.5 & 3.5 & 3.5 \\
$\xi_{1}$ & 3.2 & 2.96 & 2.96 & 2.96 & 2.768 & 2.768 \\
$\xi_{2}$ & 2.9024 & 2.57754 & 2.55296 & 2.52347 & 2.36962 & 2.33502 \\
$\xi_{3}$ & 2.66692 & 2.34146 & 2.31345 & 2.27631 & 2.17483 & 2.14147 \\
$\xi_{4}$ & 2.48921 & 2.20038 & 2.17653 & 2.14387 & 2.08208 & 2.05893 \\
$\xi_{5}$ & 2.35737 & 2.1171 & 2.09908 & 2.07434 & 2.03837 & 2.02436 \\
$\xi_{6}$ & 2.26037 & 2.06825 & 2.05548 & 2.03823 & 2.01789 & 2.01002 \\
$\xi_{7}$ & 2.18935 & 2.03971 & 2.03102 & 2.0196 & 2.00833 & 2.00028 \\
$\xi_{8}$ & 2.13752 & 2.02307 & 2.01733 & 2.01002 & 2.00387 & 2.00168 \\
$\xi_{9}$ & 2.09977 & 2.01339 & 2.00967 & 2.00512 & 2.0018 & 2.00005 \\
$\xi_{10}$ & 2.07233 & 2.00777 & 2.00539 & 2.00261 & 2.00083 & 2.00028 \\
$\xi_{11}$ & 2.0524 & 2.0045 & 2.00301 & 2.00133 & 2.00039 & 2.00011 \\
$\xi_{12}$ & 2.03794 & 2.00261 & 2.00168 & 2.00068 & 2.00018 & 2.00005 \\
$\xi_{13}$ & 2.02746 & 2.00151 & 2.00093 & 2.00034 & 2.00008 & 2.00002 \\
$\xi_{14}$ & 2.01987 & 2.00087 & 2.00052 & 2.00017 & 2.00004 & 2.00001 \\
$\xi_{15}$ & 2.01437 & 2.00051 & 2.00029 & 2.00009 & 2.00002 & 2 \\
$\xi_{16}$ & 2.01039 & 2.00029 & 2.00016 & 2.00004 & 2.00001 & 2 \\
$\xi_{17}$ & 2.00751 & 2.00017 & 2.00009 & 2.00002 & 2 & 2 \\
$\xi_{18}$ & 2.00543 & 2.0001 & 2.00005 & 2.00001 & 2 & 2 \\
$\xi_{19}$ & 2.00392 & 2.00006 & 2.00003 & 2.00001 & 2 & 2 \\
$\xi_{20}$ & 2.00283 & 2.00003 & 2.00002 & 2 & 2 & 2 \\
\hline & & & & & &
\end{tabular}

Thus, $\left\|u_{n+1}-p\right\| \leq k^{2(n+1)}\left\|\left(u_{0}-p\right)\right\| \prod_{i=0}^{n}\left\{\left(1-\vartheta_{i}\right)(1-k)\right\}$ . Now, since $\theta \leq \theta_{n} \vartheta \leq \vartheta_{n}$ and for all $n \in N$, we obtain $\| u_{n+1}$ $-p\left\|\leq k^{2(\mathrm{n}+1)}\right\|\left(u_{0}-p\right) \|\left\{1-\vartheta \theta_{i}(1-k)\right\}^{n+1}$. Let $b_{n}=k^{2(n+1)} \|($ $\left.u_{0}-p\right) \|\left\{1-\vartheta \theta_{i}(1-k)\right\}^{n+1}$.

Then,

$$
\begin{aligned}
\frac{a_{n}}{b_{n}} & =\frac{k^{3(n+1)}\left\|\left(\xi_{0}-p\right)\right\|\left\{1-\left(\theta_{i}+k \theta_{i} \vartheta_{i}\right)(1-k)\right\}^{n+1}}{k^{2(n+1)}\left\|\left(u_{0}-p\right)\right\|\left\{1-\theta_{i}(1-k)\left(1-k \vartheta_{i}\right)\right\}^{n+1}} \\
& =\frac{k^{n+1}\left\{1-\theta_{i}(1-k)\right\}^{n+1}}{\left\{1-\theta_{i}(1-k)\left(1-k \vartheta_{i}\right)\right\}^{n+1}} .
\end{aligned}
$$

Thus, taking the limit as $n \rightarrow \infty \lim _{n \rightarrow \infty}\left(b_{n} / a_{n}\right)=0$. Hence, the result follows.

Now, we prove by numerical example that our $D$ I.P has a better approximation rate than existing I.Ps in literature.

Example 10. Let a contraction mapping $F: R \rightarrow R$ be defined by $(\xi)=(4 \xi+2) / 5$. Let $\theta_{n}=2 n /(3 n+1)$ and $\vartheta_{n}=3 n /(4 n+1$ ) . The iterative values for $\xi_{0}=3.5$ are defined in Table 1 . Figures 1(a) and 1(b) present the convergence graph.

By Figures 1(a) and 1(b) and Table 1, it is clear that the new $D$ I.P has a better approximation rate then the $K, M^{*}$, $M$, Picard-S, and $S$ I.P. 

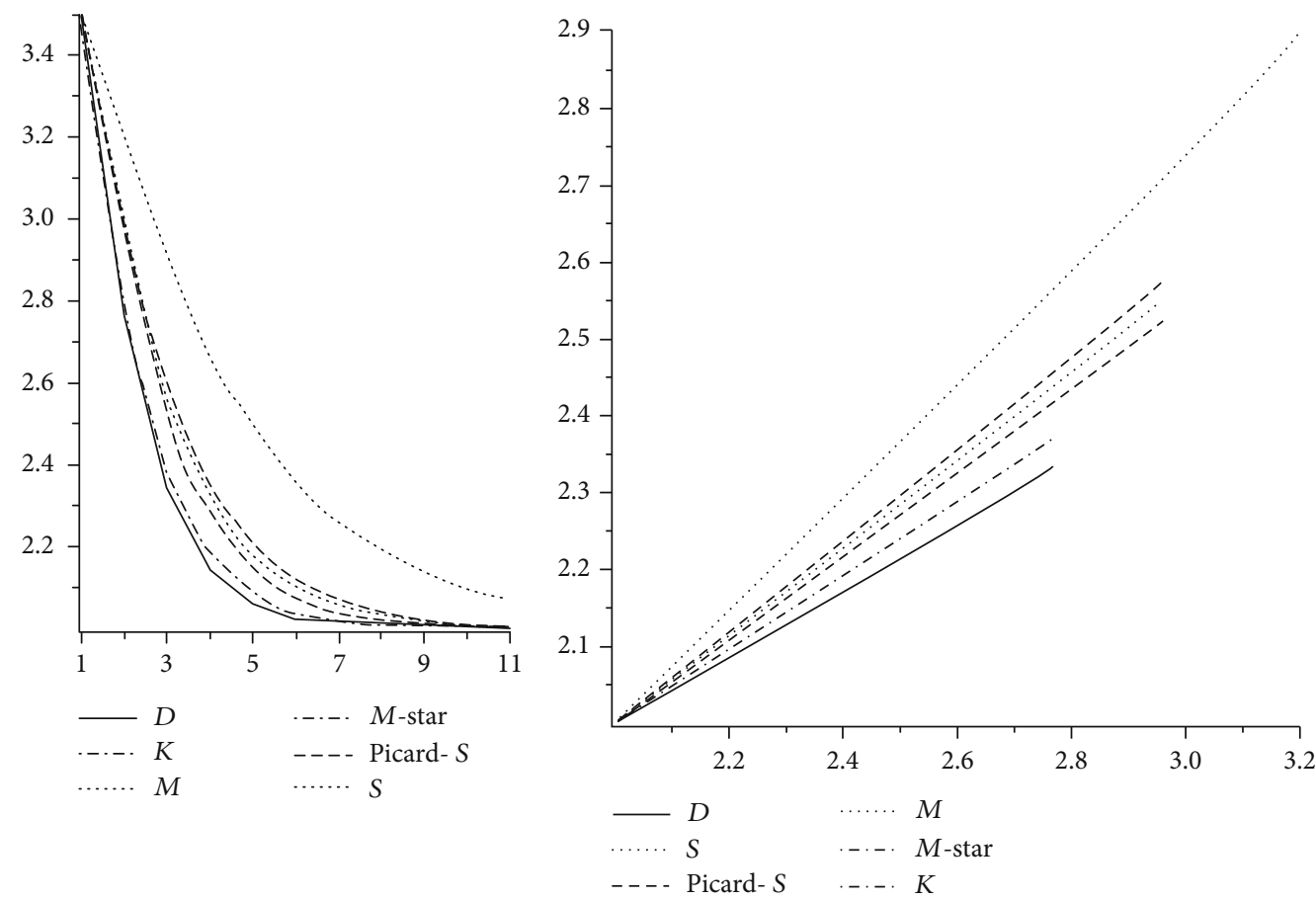

(a)

(b)

Figure 1: (a, b) Convergence of $D, K, M^{*}, M$, Picard-S, and $S$ I.P to the fixed point 2 for $F$ of Example 10.

TAble 2: Sequence generated by $D, K, M^{*}, M$, Picard-S, and $S$ I.P for $F$ of Example 11.

\begin{tabular}{|c|c|c|c|c|c|c|}
\hline & $S$ & Picard-S & $M$ & $M^{*}$ & K & $D$ \\
\hline$\xi_{0}$ & 1.5 & 1.5 & 1.5 & 1.5 & 1.5 & 1.5 \\
\hline$\xi_{1}$ & 1.732050808 & 1.981969534 & 1.981969534 & 1.98197 & 2.219882 & 2.219882 \\
\hline$\xi_{2}$ & 2.022743362 & 2.453392689 & 2.482109844 & 2.506389 & 2.727322 & 2.75395 \\
\hline$\xi_{3}$ & 2.302462597 & 2.747657664 & 2.776630296 & 2.801381 & 2.920085 & 2.936761 \\
\hline$\xi_{4}$ & 2.530373744 & 2.892112825 & 2.910917465 & 2.926554 & 2.977931 & 2.984781 \\
\hline$\xi_{5}$ & 2.69661837 & 2.955490157 & 2.965667023 & 2.973809 & 2.994026 & 2.99642 \\
\hline$\xi_{6}$ & 2.809384444 & 2.98193592 & 2.986964491 & 2.990812 & 2.998385 & 2.999166 \\
\hline$\xi_{7}$ & 2.882389407 & 2.992726282 & 2.995085112 & 2.996804 & 2.999571 & 2.999807 \\
\hline$\xi_{8}$ & 2.928277354 & 2.997083159 & 2.998153608 & 2.998893 & 2.999885 & 2.999955 \\
\hline$\xi_{9}$ & 2.956589022 & 2.998833116 & 2.9999307853 & 2.999618 & 2.999969 & 2.99999 \\
\hline$\xi_{10}$ & 2.973852778 & 2.99953392 & 2.999740915 & 2.999869 & 2.999992 & 2.999998 \\
\hline$\xi_{11}$ & 2.984301526 & 2.999814051 & 2.999903123 & 2.999955 & 2.999998 & 2.999999 \\
\hline$\xi_{12}$ & 2.990595194 & 2.999925877 & 2.999963806 & 2.999985 & 2.999999 & 3 \\
\hline$\xi_{13}$ & 2.994374126 & 2.999970477 & 2.999986488 & 2.999995 & 3 & 3 \\
\hline$\xi_{14}$ & 2.996638281 & 2.999988247 & 2.999994958 & 2.999998 & 3 & 3 \\
\hline$\xi_{15}$ & 2.997992835 & 2.999995323 & 2.99999812 & 2.999999 & 3 & 3 \\
\hline$\xi_{16}$ & 2.998802339 & 2.99999814 & 2.999999298 & 3 & 3 & 3 \\
\hline$\xi_{17}$ & 2.999285722 & 2.99999926 & 2.999999738 & 3 & 3 & 3 \\
\hline$\xi_{18}$ & 2.999574185 & 2.999999705 & 2.999999902 & 3 & 3 & 3 \\
\hline$\xi_{19}$ & 2.999746241 & 2.999999883 & 2.999999963 & 3 & 3 & 3 \\
\hline$\xi_{20}$ & 2.999848822 & 2.999999953 & 2.999999985 & 3 & 3 & 3 \\
\hline
\end{tabular}




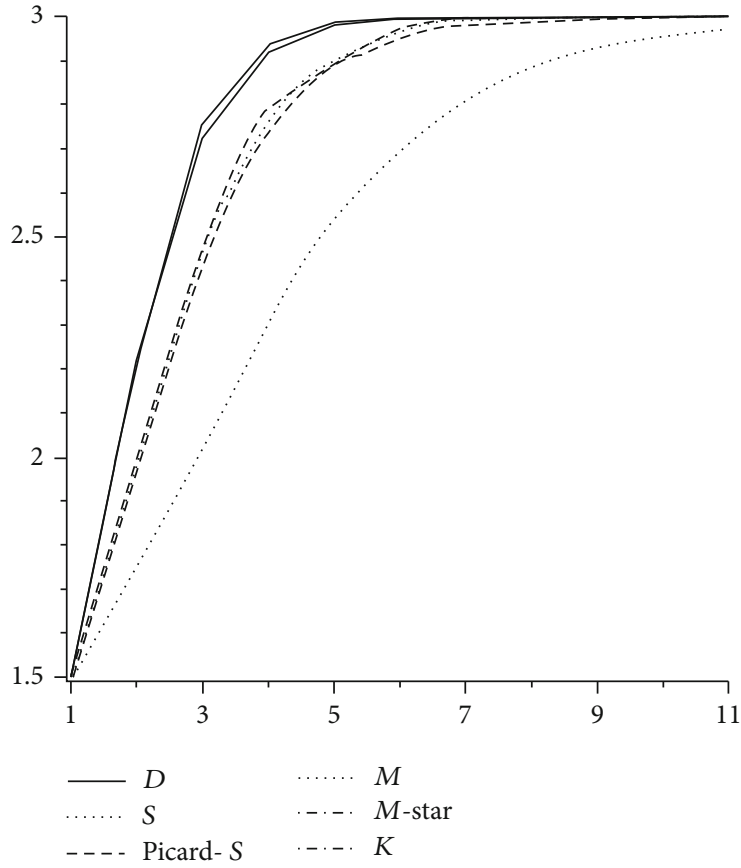

(a)

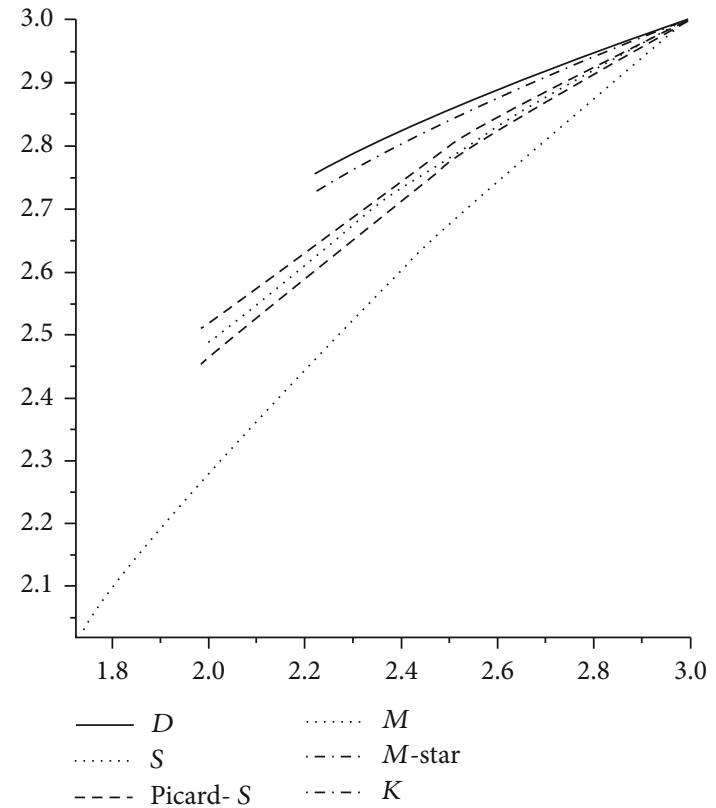

(b)

Figure 2: $(\mathrm{a}, \mathrm{b})$ Convergence of D, K, $M^{*}, \mathrm{M}$, Picard-S, S I.P to the fixed point 3 for F of Example 11.

Example 11. Let a contraction mapping $F:[0,50) \rightarrow[0,50]$ by $F(\xi)=\sqrt{4 \xi-3}$. Let $\theta_{n}=2 n /(3 n+1)$ and $\vartheta_{n}=3 n /(4 n$ $+1)$; the iterative values for the initial guess $\xi_{0}=1.5$ are given in Table 2. Figures $2(\mathrm{a})$ and $2(\mathrm{~b})$ represent the convergence graph. We can see that our new I.P (13) has a better approximation rate as compared to $S$, Picard- $S, M$, $M^{*}$, and $K$ I.P.

By Figures 2(a) and 2(b) and Table 2, it is proven that the new $D$ iteration has a better approximation rate than the $K$, $M^{*}, M$, Picard-S, and $S$ I.P.

\section{Convergence Results of a Sequence Generated by $D$ I.P}

Here, we prove some strong and weak convergence result of sequence generated by D I.P for the Suzuki generalized nonexpansive mapping in uniformly convex Banach spaces.

Lemma 12. Let $C \neq \phi \subseteq X$ and let contraction mapping $F$ $: C \rightarrow C$ be a holding condition $(M)$ and also $T(F) \neq \varnothing$. For randomly chosen $\xi_{0} \in C$, let the sequence $\left\{\xi_{n}\right\}$ be generated by (13), then $\lim _{n \rightarrow \infty}\left\|\xi_{n}-p\right\|$ exists for any $p \in T(F)$.

Proof. Let $p \in T(F)$ and $\omega \in C$. As $F$ holds the $(M)$ condition, so

$$
\frac{1}{2}\|p-F p\|=0 \leq\|p-\omega\| \Rightarrow\|F p-F \omega\| \leq\|p-\omega\| .
$$

So from 1.1(ii), we have (a) $\left\|\omega_{n}-p\right\|=\left\|\left(1-\vartheta_{n}\right) \xi_{n}+\vartheta_{n} F \xi_{n}-p\right\| \leq\left(1-\vartheta_{n}\right) \| \xi_{n}-$ $p \|+\left(\vartheta_{n}\left\|F \xi_{n}-p\right\| \leq\left(1-\vartheta_{n}\right)\left\|\xi_{n}-p\right\|+\left(\vartheta_{n}\left\|\xi_{n}-p\right\|\right.\right.$ $=\left\|\xi_{n}-p\right\|$.

So by using (a), we get

(b) $\left\|\eta_{n}-p\right\|=\left\|F\left(\left(1-\theta_{n}\right) F \xi_{n}+\theta_{n} F \omega_{n}\right)-p\right\| \leq \|\left(1-\theta_{n}\right.$ )$F \xi_{n}+\theta_{n} F \omega_{n}-p \leq\left(1-\theta_{n}\right)\left\|F \xi_{n}-p\right\|+\theta_{n} \| F \omega_{n}-p$ $\| \leq\left(1-\theta_{n}\left\|\xi_{n}-p\right\|+\theta_{n}\left\|\omega_{n}-p\right\| \leq\left(1-\theta_{n}\right)\left\|\xi_{n}-p\right\|\right.$ $+\theta_{n}\left\|\xi_{n}-p\right\|=\left\|\xi_{n}-p\right\|$.

So using (b), we have

(c) $\left\|\xi_{n+1}-p\right\|=\left\|F \eta_{n}-p\right\| \leq\left\|\eta_{n}-p\right\| \leq\left\|\xi_{n}-p\right\|$,

which means that $\left\|\xi_{n}-p\right\|$ is nonincreasing and bounded, $\forall p \in T(F)$. So $\lim _{n \rightarrow \infty}\left\|\xi_{n}-p\right\|$ exists, hence proven.

Theorem 13. Let $C \neq \phi \subseteq X$, and let contraction mapping $F$ $: C \rightarrow C$ holding condition $(M)$. For randomly chosen $\xi_{0} \in C$, let $\left\{\xi_{n}\right\}$ be the sequence generated by (13) $\forall n \geq 1$ where $\theta_{n}$ and $\vartheta_{n}$ are sequences of real numbers $\in[01]$. Then, $T(F) \neq \varnothing$ iff $\xi_{n}$ is bounded and $\lim _{n \rightarrow \infty}\left\|F \xi_{n}-\xi_{n}\right\|=0$.

Proof. Assume $F(T) \neq \phi$ and consider $p \in F(T)$. So by result 3.1, $\lim _{n \rightarrow \infty}\left\|\xi_{n}-p\right\|$ exists and $\xi_{n}$ is bounded put

(d) $\lim _{n \rightarrow \infty}\left\|\xi_{n}-p\right\|=r$.

From (a) and (d), we have

(e) $\lim _{n \rightarrow \infty} \sup \left\|\omega_{n}-p\right\| \leq \lim _{n \rightarrow \infty} \sup \left\|\xi_{n}-p\right\|=r$. 
By result 1.1(ii), we have

(f) $\lim _{n \rightarrow \infty} \sup \left\|T \xi_{n}-p\right\| \leq \lim _{n \rightarrow \infty} \sup \left\|\xi_{n}-p\right\|=r$.

On the other hand,

$$
\begin{aligned}
\left\|\xi_{n+1}-p\right\| & =\left\|F \eta_{n}-p\right\| \leq\left\|\eta_{n}-p\right\| \\
& =\left\|F\left(\left(1-\theta_{n}\right) F \xi_{n}+\theta_{n} F \omega_{n}\right)-p\right\| \\
& \leq\left\|\left(1-\theta_{n}\right) F \xi_{n}+\theta_{n} F \omega_{n}-p\right\| \\
& \leq\left(1-\theta_{n}\right)\left\|F \xi_{n}-p\right\|+\theta_{n}\left\|F \omega_{n}-p\right\| \\
& \leq\left(1-\theta_{n}\left\|\xi_{n}-p\right\|+\theta_{n}\left\|\omega_{n}-p\right\|\right. \\
& \leq\left\|\xi_{n}-p\right\|-\theta_{n}\left\|\xi_{n}-p\right\|+\theta_{n}\left\|\omega_{n}-p\right\| .
\end{aligned}
$$

This implies that

$$
\frac{\left\|\xi_{n+1}-p\right\|-\left\|\xi_{n}-p\right\|}{\theta_{n}} \leq\left\|\omega_{n}-p\right\|-\left\|\xi_{n}-p\right\| .
$$

So

$$
\begin{aligned}
\left\|\xi_{n+1}-p\right\|-\left\|\xi_{n}-p\right\| & \leq \frac{\left\|\xi_{n+1}-p\right\|-\left\|\xi_{n}-p\right\|}{\theta_{n}} \\
& \leq\left\|\omega_{n}-p\right\|-\left\|\xi_{n}-p\right\|
\end{aligned}
$$

implies that

$$
\left\|\xi_{n+1}-p\right\| \leq\left\|\omega_{n}-p\right\|
$$

Therefore,

(g) $r \leq \lim _{n \rightarrow \infty} \inf \left\|\omega_{n}-p\right\|$

From (e) and (g), we get

(h) $r=\lim _{n \rightarrow \infty}\left\|\omega_{n}-p\right\|=\lim _{n \rightarrow \infty} \|\left(1-\vartheta_{n}\right) \xi_{n}+\vartheta_{n} F \xi_{n}-$ $p\left\|=\lim _{n \rightarrow \infty}\right\|\left(\vartheta_{n}\right)\left(F \xi_{n}-p\right)+\left(1-\vartheta_{n}\right)\left(\xi_{n}-p\right)$.

From (d), (f), and (h) together with Lemma 4, we have

$$
\lim _{n \rightarrow \infty}\left\|F \xi_{n}-\xi_{n}\right\|=0 .
$$

On the other hand, assume that $\xi_{n}$ is a bounded sequence and $\lim _{n \rightarrow \infty}\left\|F \xi_{n}-\xi_{n}\right\|=0$. Also, $p \in A\left(C, \xi_{n}\right)$. So by Proposition 1 ,

$$
\begin{aligned}
r\left(F p, \xi_{n}\right) & =\lim _{n \rightarrow \infty} \sup \left\|\xi_{n}-F p\right\| \\
& \leq \lim _{n \rightarrow \infty} \sup \left(3\left\|F \xi_{n}-\xi_{n}\right\|+\left\|\xi_{n}-p\right\|\right) \\
& \leq \lim _{n \rightarrow \infty} \sup \left\|\xi_{n}-p\right\|=r\left(p, \xi_{n}\right) .
\end{aligned}
$$

Hence, $F p \in A\left(C, \xi_{n}\right)$. As $X$ is uniformly convex, and $A($ $\left.C, \xi_{n}\right)$ is a singleton set, therefore $F p=p$. Hence, $(F) \neq \varnothing$.

\subsection{Weak and Strong Convergence Theorem}

Theorem 14. Let $C \neq \phi \subseteq X$, where $X$ satisfies Opial condition, and let $F: C \rightarrow C$ holding condition $(M)$, where $\theta_{n}$ and $\vartheta_{n}$ are real sequences $\epsilon[01]$, such that $T(F) \neq \varnothing$. For randomly selected $\xi_{0} \in C$, consider $x_{n}$ to be the sequence generated by (13) $\forall n \geq 1$. Then, the sequence generated by the $D$ iterative process converges weakly to $p \in T(F)$.

Proof. As $T(F) \neq \phi$, and from Theorem $13, \xi_{n}$ is a bounded sequence and $\lim _{n \rightarrow \infty}\left\|F \xi_{n}-\xi_{n}\right\|=0$. Being uniformly convex $X$ must be reflexive, so by Eberlin's theorem $\exists$, a subsequence $\xi_{n_{j}}$ of $\xi_{n}$ which approaches weakly to $p_{1} \in X$. As $C$ is closed and convex, by Mazur's theorem, $p_{1} \in C$. By Lemma 2, $p_{1} \in T(F)$. Next, we prove that $\xi_{n}$ approaches weakly to $p_{1}$. Actually, if it is not true, so $\exists$ a subsequence $\xi_{n_{k}}$ for $\xi_{n}$ s.t $\xi_{n_{k}}$ converges weakly to $p_{2} \in C$ and $p_{2} \neq p_{1}$. By Lemma 2, $p_{2} \in T(F)$. Since $\lim _{n \rightarrow \infty}\left\|\xi_{n}-p\right\|$ defined $\forall p \in T(F)$. Thus, from Theorem 13 and Opial's property,

$$
\begin{aligned}
\lim _{n \rightarrow \infty}\left\|\xi_{n}-p_{1}\right\| & =\lim _{j \rightarrow \infty}\left\|\xi_{n_{j}}-p_{1}\right\|<\lim _{j \rightarrow \infty}\left\|\xi_{n_{j}}-p_{2}\right\| \\
& =\lim _{n \rightarrow \infty}\left\|\xi_{n}-p_{2}\right\|=\lim _{k \rightarrow \infty}\left\|\xi_{n_{k}}-p_{2}\right\| \\
& <\lim _{k \rightarrow \infty}\left\|\xi_{n_{k}}-p_{1}\right\|=\lim _{n \rightarrow \infty}\left\|\xi_{n}-p_{1}\right\|,
\end{aligned}
$$

which is a contradiction to fact. So $p_{1}=p_{2} \Rightarrow \xi_{n}$ converges weakly to a single fixed point of $F$.

Next, we prove the strong convergence theorem.

Theorem 15. Let $C \neq \phi \subseteq X$, and let contraction mapping $F$ $: C \rightarrow C$ holding condition $(M)$, where $\theta_{n}$ and $\vartheta_{n} \in\left[\begin{array}{ll}0 & 1\end{array}\right]$ are real sequences, s.t $T(F) \neq \varnothing$. Then, $\xi_{n}$ approaches strongly to $p \in T(F)$.

Proof. By Lemma 3, $T(F) \neq \phi$, and by Theorem 13, we have $\lim _{n} \rightarrow \infty\left\|T \xi_{n}-\xi_{n}\right\|=0$. As $C$ is compact, so $\exists$ a subsequence $\xi_{n_{j}}$ of $\xi_{n}$ which approaches strongly to $p$ where $p \in$ $C$. By Proposition 1 (iii), we have $\left\|\xi_{n_{k}}-T p\right\| \leq 3\left\|T \xi_{n_{k}}-\xi_{n_{k}}\right\|$ $+\left\|\xi_{n_{k}}-p\right\|, \forall n \geq 1$. Letting $k \rightarrow \infty$, we attain $p \in T(F)$. As, by Lemma $12, \lim _{n} \rightarrow\left\|\xi_{n}-p\right\|$ has a defined value, for every $p \in F(T)$, so $\xi_{n}$ converge strongly to $p$.

\section{Conclusion}

In this article, we present the new fastest iteration method to approximating fixed point of contraction mapping. First, we present the $D$ iteration process and prove weak and strong convergence results. Also, we analytically and numerically proved that the $D$ iteration process has a better approximation rate than existing iteration processes as defined in [1$4,6,7,9]$.

\section{Data Availability}

No data use for this study.

\section{Conflicts of Interest}

The writers announce that they do not have any competing interests. 


\section{Authors' Contributions}

All authors contributed fairly and significantly in writing this article. All authors have read and agreed to the published version of the manuscript.

\section{Acknowledgments}

The authors give thanks to their universities.

\section{References}

[1] R. P. Agarwal, D. O. Regan, and D. R. Sahu, "Iterative construction of fixed points of nearly asymptotically nonexpansive mappings," Journal of Nonlinear and convex Analysis, vol. 8, pp. 61-79, 2007.

[2] V. Berinde, Iterative Approximation of Fixed Points, Springer, Berlin, 2007.

[3] S. H. Khan, "A Picard-Mann hybrid iterative process," Fixed Point Theory Application, vol. 2013, no. 1, p. 69, 2013.

[4] Z. Opial, "Weak convergence of the sequence of successive approximations for nonexpansive mappings," Bulletin of the American Mathematical Society, vol. 73, pp. 595-597, 1967.

[5] B. S. Thakur, D. Thakur, and M. Postolache, "A new iterative scheme for numerical reckoning fixed points of Suzuki's generalized nonexpansive mappings," Applied Mathematics and Computation, vol. 275, pp. 147-155, 2016

[6] K. Ullah and M. Arshad, "New iteration process and numerical reckoning fixed points in Banach spaces," University Politehnica of Bucharest Scientific Bulletin Series A, vol. 79, no. 4, pp. 113-122, 2017.

[7] K. Ullah and M. Arshad, "Numerical reckoning fixed points for Suzuki's generalized nonexpansive mappings via new iteration process," Filomat, vol. 32, pp. 187-196, 2018.

[8] A. Hussain, D. Ali, and E. Karapinar, "Stability data dependency and errors estimation for a general iteration method," Alexandria Engineering Journal, vol. 60, no. 1, pp. 703-710, 2021.

[9] M. Abbas and T. Nazir, "A new faster iteration process applied to constrained minimization and feasibility problems," Matematicki Vesnik, vol. 66, pp. 223-234, 2014.

[10] N. Hussain, K. Ullah, and M. Arshad, "Fixed point approximation of Suzuki generalized nonexpansive mappings via new faster iteration process," vol. 19, no. 8, pp. 1383-1393, 2018.

[11] F. Gursoy and V. Karakaya, "A Picard-S hybrid type iteration method for solving a differential equation with retarded argument," 2014, https://arxiv.org/abs/1403.2546.

[12] T. Suzuki, "Fixed point theorems and convergence theorems for some generalized nonexpansive mappings," Journal of Mathematical Analysis and Applications, vol. 340, no. 2, pp. 1088-1095, 2008.

[13] J. Schu, "Weak and strong convergence to fixed points of asymptotically nonexpansive mappings," Bulletin of the Australian Mathematical Society, vol. 43, no. 1, pp. 153-159, 1991.

[14] K. Goebel and W. A. Kirk, Topic in Metric Fixed Point Theory Application, Cambridge Universty Press, 1990.

[15] W. R. Mann, "Mean value methods in iteration," Proceedings of American Mathematical Society, vol. 4, no. 3, pp. 506-510, 1953.

[16] S. Suantai, W. Cholamjiak, and W. Cholamjiak, "An implicit iteration process for solving a fixed point problem of a finite family of multi-valued mappings in Banach spaces," Applied Mathematics Letters, vol. 25, no. 11, pp. 1656-1660, 2012.

[17] P. Cholamjiak and S. Suantai, "Weak convergence theorems for a countable family of strict pseudocontractions in Banach spaces," Fixed Point Theory and Applications, vol. 2010, Article ID 632137, 16 pages, 2010.

[18] P. Sunthrayuth and P. Cholamjiak, "Iterative methods for solving quasi-variational inclusion and fixed point problem in quniformly smooth Banach spaces," Numerical Algorithms, vol. 78, no. 4, pp. 1019-1044, 2018. 